\title{
Adaptive Engine Torque Compensation with Driveline Model
}

\author{
Jinrak Park ${ }^{1}$, Seibum $\mathrm{Choi}^{1, \mathrm{a}}$ and Jiwon $\mathrm{Oh}^{2}$ \\ ${ }^{1}$ KAIST, Mechanical Engineering Department, 34141 Daejeon, Korea \\ ${ }^{2}$ Hyundai motor group, Research \& Development Division, 150, Hyundaiyeonguso-ro, Namyang-eup, Hwaseong-si, 18280 Gyeonggi-do, \\ Korea
}

\begin{abstract}
Engine net torque is the total torque generated by the engine side, and includes the fuel combustion torque, the friction torque, and additionally the starter motor torque in case of hybrid vehicles. The engine net torque is utilized to control powertrain items such as the engine itself, the transmission clutch, also the engine clutch, and it must be accurate for the precise powertrain control. However, this net torque can vary with the engine operating conditions like the engine wear, the changes of the atmospheric pressure and the friction torque. Thus, this paper proposes the adaptive engine net torque compensator using driveline model which can cope with the net torque change according to engine operating conditions. The adaptive compensator was applied on the parallel hybrid vehicle and investigated via MATLAB Simcape Driveline simulation.
\end{abstract}

\section{Introduction}

Recently, eco-friendly automobile technology is attracting attention. So, the automotive precision control technology is developing with electronic control system to satisfy the fuel economy and the performance of a vehicle. The accurate monitoring of operating states of a car is necessary for the precise control but sometimes it is impossible to utilize some sensors due to the lack of cost and space. Thus, the use of state observers using physical models is increasing. Typically, torque sensors are expensive and bulky, so they cannot be mounted on commercial vehicles. Thus, all torque states such as engine torque, engine clutch torque, transmission torque, motor torque, etc. should be estimated using other sensors.

Engine net torque is the torque measured at the crankshaft flywheel and is the sum of the fuel combustion torque, the friction torque and, additionally in case of hybrid vehicles, the starter motor torque. This net torque is the important state for the powertrain control such as the control of engine itself, engine clutch, transmission clutch, and for the vehicle dynamics control like active cruise control. However, the net torque cannot be measured because torque sensors cannot be utilized. So, it should be estimated. The fuel combustion torque of an engine is typically calculated through a map with the throttle opening and the engine speed. However, the mapbased state estimator cannot correspond to the change of plant model and operating condition. The fuel combustion torque can vary with the personal structure tuning, the engine wear (aging), atmospheric pressure, etc. Also, the friction torque can change depending on the condition of oil and the engine wear. And, it should be also estimated. The engine net torque can be estimated by

\footnotetext{
a Corresponding author: sbchoi@kaist.ac.kr
}

summing the fuel combustion torque and the friction torque. In case of hybrid vehicles, the starter motor torque is added to the engine net torque and it can be estimated somewhat precisely using the current sensor. However, various factors can affect the error of the engine net torque, so it is difficult to find out the cause of the error.

S. B. Choi, and J. K. Hedrick proposed the fuel combustion torque model which is accurate in both the steady and transient state [1]. Also, the engine net torque is compensated using the load torque which is assumed to be known, and driveline model. However, in commercial vehicles, it is difficult to know the exact load torque because the air drag force, the rolling resistance and the gradient resistance cannot be exactly estimated. Thus, another solution is needed to compensate the engine net torque instead of the method using the load torque.

J. Oh, J. Kim, and S. B. Choi suggested the adaptive estimator to estimate the quantity of the engine net torque compensation and the output shaft torque simultaneously using only the driveline model [2]. However, the convergent speed of estimated states could be slow because two unknown states are esimtated using only one erorr variable.

This paper proposes the adaptive engine net torque compensator using the torque compliance model of the output shaft and the driveline model to compensate the slowly varying torque error depending on the engine model change. The compensator in this paper estimates only one unknown state, the multiplication factor on the engine net torque, using one error variable. The torque compliance model of a shaft is widely used to estimate the shaft torque but it needs rotational angle and speed information of a shaft which is not suitable for a real 


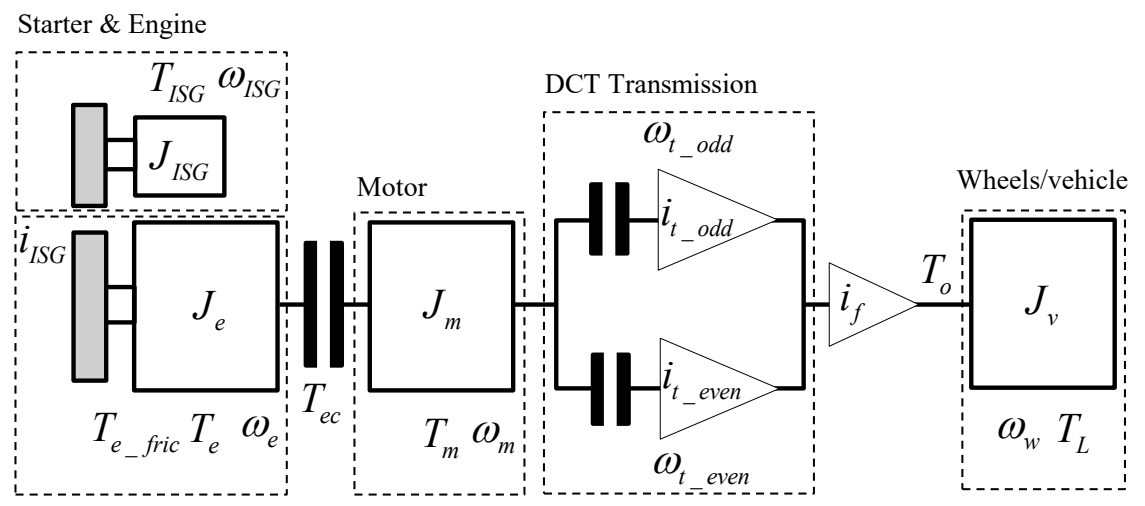

Figure 1. Driveline model of a parallel hybrid vehicle ( $J$ : inertia, $T$ : torque, $\omega$ : angular velocity, $i$ : gear ratio)

vehicle [3]. In case of using only speed information for commercial vehicles, the shaft torque is expressed as the differential form. Then, the drift issue can occur in estimating the shaft torque by integration. Thus, this paper also proposes the method to compensate the engine net torque while resolving drift issue on the shaft torque.

Also, the adaptive compensator is designed to use only sensors which can be utilized in a commercial vehicle. The adaptive algorithm is applied on a parallel hybrid vehicle in this paper but the algorithm can be modified easily for other types of a vehicle. The algorithm is investigated through MATLAB Simscape Driveline simulation. The organization of the paper is as follows.

Section 2 explains the detail algorithm of the compensator. Section 3 shows the simulation result. And, section 4 deals with the conclusion.

\section{Detail algorithm of adaptive engine net torque compensator}

\subsection{Driveline model}

Fig. 1 shows the lumped driveline model of a parallel hybrid vehicle and describes the torque flow. The mathematical model of a parallel hybrid vehicle which is utilized in this paper is as follows [4].

$$
\begin{gathered}
J_{e} \dot{\omega}_{e}=T_{e_{-} \text {net }}-T_{e c} \\
J_{m} \dot{\omega}_{m}=T_{e c}+T_{m}-\frac{T_{o}}{i_{t}} \\
\dot{T}_{o}=k_{o}\left(\frac{\omega_{t}}{i_{t}}-i_{f} \omega_{w}\right)
\end{gathered}
$$

$T_{e c}, T_{o}, k_{o}, i_{t}$, and $i_{f}$ mean the engine clutch torque, the output shaft torque, the equivalent torsional stiffness of the output shaft, the transmission gear ratio, and the final gear ratio.

The engine net torque is the sum of the fuel combustion torque, the starter motor torque, and the engine friction torque like below.

$$
T_{e_{-} \text {net }}=T_{e}+T_{I S G}-T_{e_{-} \text {fric }}
$$

$T_{I S G}$, and $T_{e \text { fric }}$ mean the starter motor torque, and the engine friction torque.

\subsection{Adaptive engine net torque compensation algorithm}

Using the mathematical driveline model of the parallel hybrid vehicle, the following equation can be reached by altering and summing (1) and (2).

$$
T_{e_{-} \text {net }}=\frac{T_{o}}{i_{t}}-T_{m}+J_{e} \dot{\omega}_{e}+J_{e} \dot{\omega}_{m}
$$

$T_{e \text { net }}$ indicates the actual engine net torque generated by the engine side.

Since the actual vehicle uses only the angular velocity sensor, the output shaft torque is expressed as a differential form as shown in equation (3), and drift occurs when the output shaft torque is obtained by integration. Thus, the torque variation formula in some specific period is utilized for the engine net torque compensation algorithm to escape from the drift issue like below.

$$
\Delta T_{e_{-} n e t}=\frac{\Delta T_{o}}{i_{t}}-\Delta\left(T_{m}+J_{e} \dot{\omega}_{e}+J_{e} \dot{\omega}_{m}\right)
$$

Assuming that the multiplied uncertainty exist on the nominal engine net torque, the engine net torque change can be expressed like below.

$$
\begin{aligned}
\hat{T}_{e_{-} n e t} & =\hat{C} T_{e_{-} n e t}^{\prime} \\
\Delta \hat{T}_{e_{-} n e t} & =\hat{C} \Delta T_{e_{-} n e t}^{\prime}
\end{aligned}
$$

$T_{e_{-} \text {net }}$, and $C$ mean the nominal engine net torque and the torque constant, and $\Delta$ indicates the amount of variation.

Then, the adaptive error is defined like below.

$$
\begin{aligned}
\varepsilon & =\Delta T_{e_{-} n e t}-\Delta \hat{T}_{e_{-} n e t}=(C-\hat{C}) \Delta T_{e_{-} n e t}^{\prime} \\
& =\Delta\left(\frac{T_{o}}{i_{t}}-T_{m}+J_{e} \dot{\omega}_{e}+J_{e} \dot{\omega}_{m}\right)-\hat{C} \Delta T_{e_{-} \text {net }}^{\prime}
\end{aligned}
$$

The adaptive law of the torque constant can be defined using the simple gradient adaptive method like below equation [5].

$$
\dot{\hat{C}}=\gamma \varepsilon \Delta T_{e_{-} \text {net }}^{\prime}(\gamma>0)
$$

Defining the torque constant error like below,

$$
\tilde{C}=C-\hat{C}
$$

The following equation is satisfied using (10) and (11). 


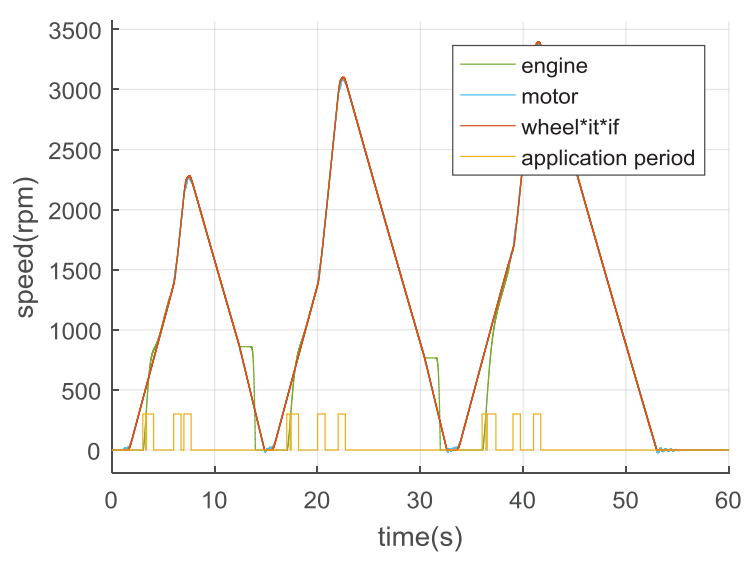

Figure 2. Rotational speed during simulation

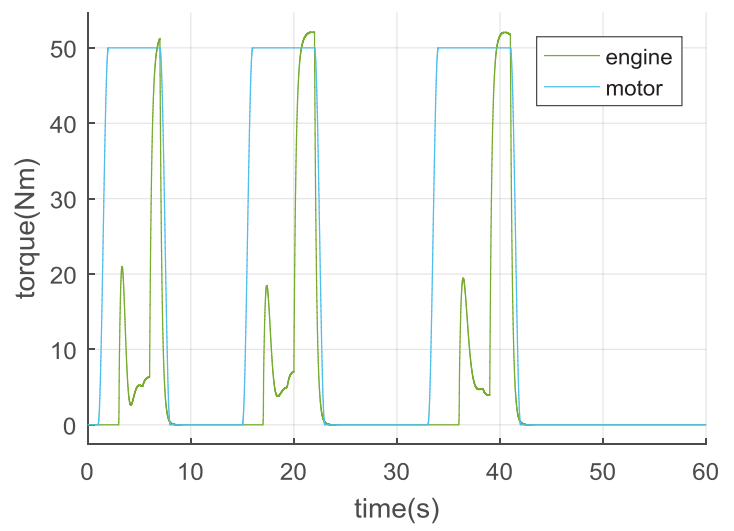

Figure 3. Torque during simulation

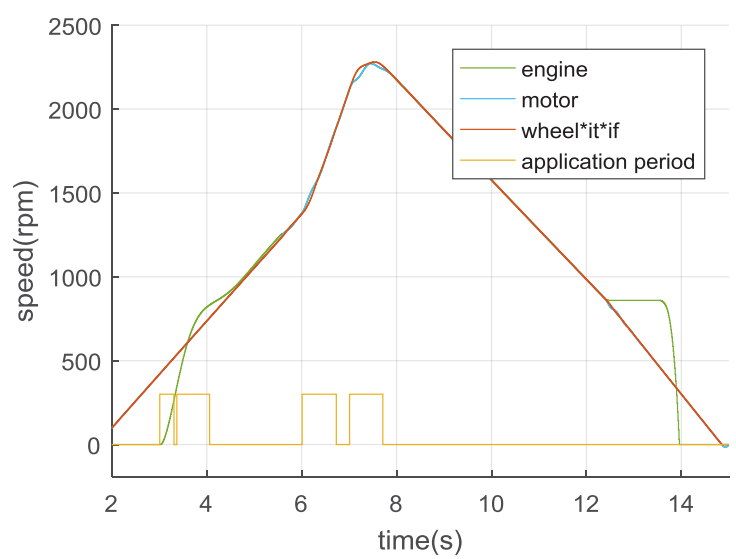

Figure 4. Enlarged graph of rotational speed during simulation

$$
\dot{\tilde{C}}=-\gamma \tilde{C} \Delta T_{e_{-} \text {net }}^{\prime}(\gamma>0)
$$

Then, the stability of the error dynamics can be proven using the simple Lyapunov candidate like below.

$$
V(\tilde{C})=\frac{\tilde{C}^{2}}{2 \gamma}
$$

$$
\dot{V}=-\tilde{C}^{2} \Delta T_{e_{-} n e t}^{\prime}{ }^{2} \leq 0
$$

Since the variation of the nominal engine net torque satisfies the PE (persistence of excitation) condition, the adapted torque constant converges to the actual torque constant.

\section{Simulation result}

\subsection{Simulation condition}

The simulation in this paper was conducted using the Simscape Driveline tool in MATLAB Simulink. Fig. 2 shows the rotational engine speed of engine, motor, wheel speed which is multiplied the transmission and final gear ratio and, the application period of the adaptive algorithm. As mentioned in the section 2, the torque variation formula was utilized to address the drift problem of the output shaft torque in some specific period. Fig. 3 shows the generated torque by the engine side (engine net torque) and the motor. Also, Fig. 4 is the enlarged plot for some seconds of Fig. 2. For this simulation, it was assumed that the engine net torque is known but has some multiplied uncertainty and, the motor speed is the same with the transmission input shaft speed. Also, during the simulation period, the transmission clutch was engaged all the time and gear shift does not happened. Also, the engine and motor speed was synchronized three times.

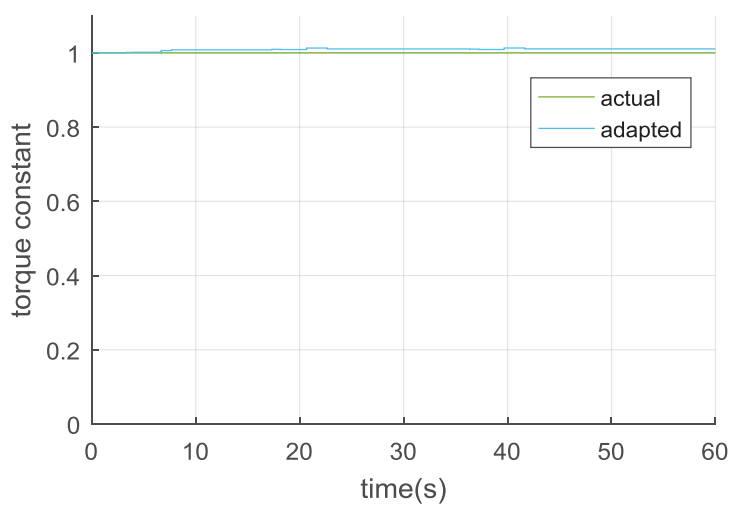

Figure 5. Result of torque compensator when the nominal torque constant is 1

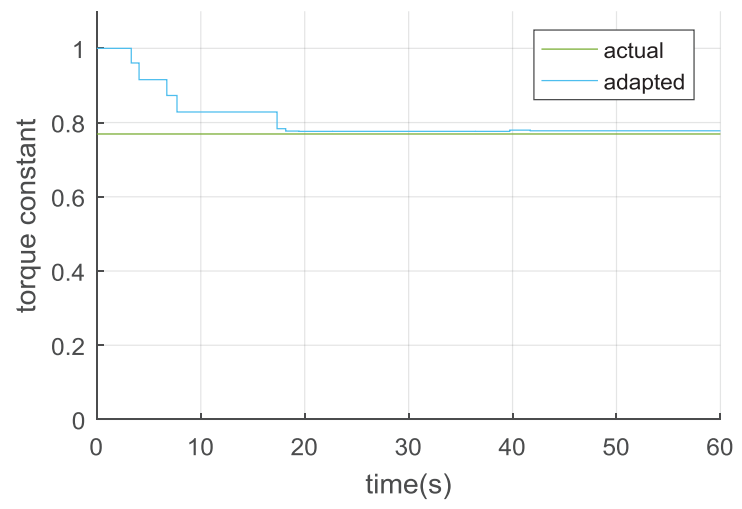

Figure 6. Result of torque compensator when the nominal torque constant is 0.77 


\section{Conclusions}

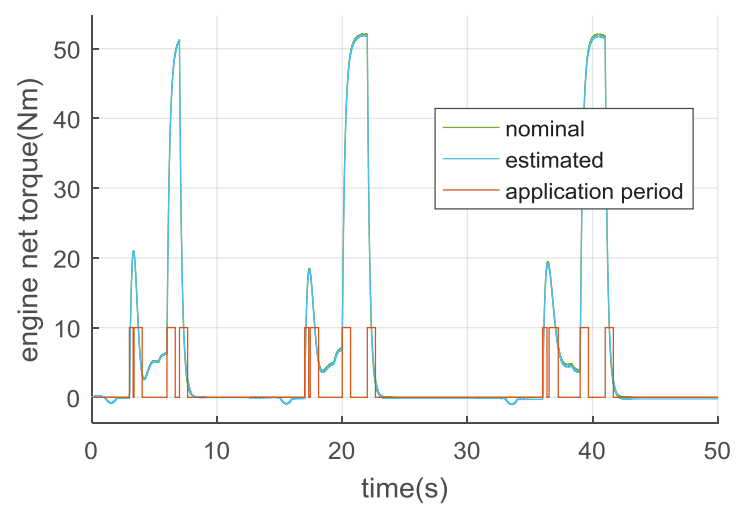

Figure 7. Comparison of engine net torque when torque constant is 1

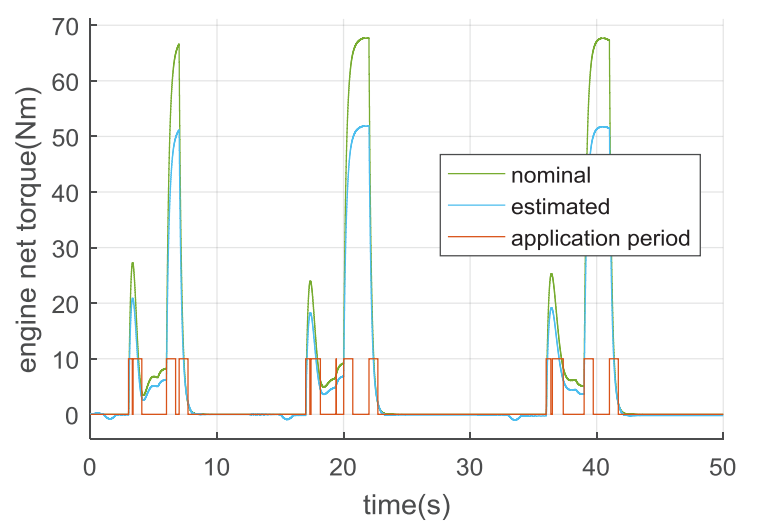

Figure 8. Comparison of engine net torque when torque constant is 0.77

\subsection{Result}

Fig. 5 shows the result of the torque compensator when the nominal torque constant is 1 . Also, Fig. 6 shows the result when the nominal torque constant is 0.77 . For the performance of the adaptive compensator, the adapted torque constant well converged to the actual torque constant but a little error, around 0.01 , existed between the actual and adapted torque constant. It is because of the error on the output shaft torque. The torque compliance model of the output shaft is expressed in the differential form so the phase lag due to the integration existed. Also, the damping term was ignored in the compliance model.

Additionally, Fig. 7 shows the nominal and estimated engine net torque during the simulation when the nominal torque constant is 1 . And, Fig. 8 shows the same thing when the nominal torque constant is 0.77 . As you can see in Fig. 7 and Fig. 8, the adaptive torque compensator compares the difference of the nominal and estimated engine net torque, respectively, for some specific periods, and the torque constant is adapted using that difference error.
This paper proposed the adaptive engine net torque compensator according to the multiplied uncertainty on the net torque. This is to compensate the net torque error which is caused by the slow variation of the engine model. The engine model can change when the engine ages or wears, and can also be changed by an external environment, such as atmospheric pressure. The adaptive law of the torque constant for the engine net torque compensator was designed based on the variation of the engine net torque. The adaptive laws for the torque compensator was investigated through the simulation using the Simscape Driveline tool in MATLAB. The error between the actual and adapted torque constant occurred around in amount of 0.01. It is because of the error on the estimated output shaft torque. The improvement of this problem is left as a future work.

\section{References}

1. S. B. Choi, and J. K. Hedrick. "Robust throttle control of automotive engines: Theory and experiment." Journal of dynamic systems, measurement, and control, 118.1, 92-98, (1996)

2. J. Oh, J. Kim, and S. B. Choi. "Design of estimators for the output shaft torque of automated manual transmission systems. " In Industrial Electronics and Applications (ICIEA), 2013 8th, 1370-1375, (2013, June).

3. J. Oh, and S. B. Choi. "Real-time estimation of transmitted torque on each clutch for ground vehicles with dual clutch transmission." IEEE/ASME Transactions on Mechatronics, 20(1), 24-36, (2015)

4. J. Oh, S. B. Choi, Y. J. Chang, and J. S. Eo. "Engine clutch torque estimation for parallel-type hybrid electric vehicles." International Journal of Automotive Technology, 18(1), 125-135, (2017).

5. P. A. Ioannou, \& J. Sun. "Robust adaptive control." Upper Saddle River, NJ: PTR Prentice-Hall, 1, (1996)

\section{Acknowledgement}

This work was supported in parts by the National Research Foundation of Korea grant funded by the Korea government (MSIP) (No.2017R1A2B4004116), the Ministry of Science and ICT, Korea, under the Information Technology Research Center support program (IITP-2017-2012-0-00628) supervised by the Institute for Information and communications Technology Promotion, and the BK21 Plus Program. 